\title{
Lifestyle-related Factors in Stroke and Dementia
}

\author{
a report by \\ Miia Kivipelto ${ }^{1}$ and Alina Solomon ${ }^{2}$ \\ 1. Ageing Research Center, Department of Neurobiology, Caring Sciences and Society, Karolinska Institute, Stockholm;
}

2. Department of Neurology, University of Kuopio

DOI:10.17925/ENR.2008.03.01.52

The current literature available on databases in medicine and related areas clearly shows a remarkable growth of knowledge in neuro-degenerative and cerebrovascular diseases. However, it shows an equally remarkable gap between the 'neurodegenerative field' and the 'vascular field'. Research on dementia/cognitive impairment is heavily 'alzheimerised', giving vascular pathologies secondary status. Research on stroke/cerebrovascular disorders has a tendency to leave out cognition. This separation has led to two main lines of studies, focused on two extremes: clinical stroke and dementia. Both stroke and dementia are common in older age, so their joint occurrence is not a rare phenomenon. An examination of the literature of related disciplines shows that stroke and dementia actually have more in common than was previously thought.

Outcomes in epidemiological studies, stroke and dementia are difficult to define, as both are heterogeneous and multifactorial conditions with genetic and environmental risk factors. Most epidemiological studies do not have reliable information about stroke/dementia types and the mechanisms involved. Stroke is usually classified as ischaemic or haemorrhagic, but the aetiology is far more heterogeneous: approximately $50 \%$ of ischaemic strokes, for example, are caused by large-artery atherosclerosis, $25 \%$ by small-vessel disease, $20 \%$ by cardiac embolism and $5 \%$ by other causes, e.g. arterial dissection. ${ }^{1}$ Various subtypes of ischaemic stroke may have different risk factors, e.g. atrial fibrillation and mitral valve disease are related to cardioembolic stroke, but not to atherosclerotic stroke. A history or indication of recent stroke is included in the diagnostic criteria for vascular dementia $(\mathrm{VaD})$, so risk factors for $\mathrm{VaD}$ are presumed to be similar to those of cerebrovascular disease. However, to complicate matters, cerebrovascular lesions often co-exist and interact with neurodegenerative changes, playing an important role in dementia of Alzheimer's disease (AD). The co-existence of $A D$ type and vascular changes seems to be more frequent than would be expected by chance, ${ }^{2,3}$ and $A D$ and $V a D$ have a significant overlapping of risk factors, clinical features and pathology. 'Pure' $A D$ and $\mathrm{VaD}$ can be considered opposite ends of a dementia aetiology continuum, in which most cases have combinations of $A D$ type and vascular changes of different degrees. ${ }^{4}$ Epidemiological studies with stroke or dementia (i.e. clinical extremes) as outcomes merely see the tip of the iceberg. Subclinical vascular disease (e.g. silent infarcts, microhaemorrhages) is more frequent than clinical stroke, ${ }^{5}$ and can lead to subtle cumulative brain damage over time,

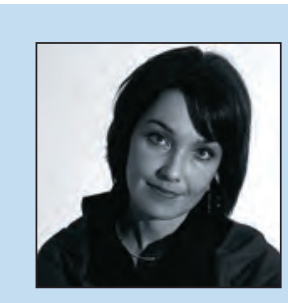

Miia Kivipelto is an Associate Professor of Neurology/ Neuroepidemiology at the Ageing Research Centre, Karolinska Institutet, and at the Department of Neurology, University of Kuopio. She also works as a physician at the Memory Clinic, Karolinska University Hospital. Dr Kivipelto is the principal investigator of the Cardiovascular Risk Factors, Aging and Dementia (CAIDE) study.

E: miia.kivipelto@ki.se increasing the risk of clinical stroke and dementia ( $A D, V a D$ or mixed). $A D$ has a long pre-clinical phase, with neuropathological changes starting decades before the disease becomes clinically manifest as cognitive impairment/dementia. The concepts of vascular cognitive impairment (VCI) and mild cognitive impairment $(\mathrm{MCl})$ are attempts to refine outcomes and define groups of individuals who may benefit more from interventional strategies. For example, patients with cognitive impairment are often excluded/not identified in stroke clinical trials. In confronting stroke and dementia, the best time for preventative interventions would be the 'brain at risk' stage before clinical manifestations appear. ${ }^{5}$

The purpose of this article is to give a brief overview of lifestyle-related factors in stroke and dementia, with an emphasis on integration instead of separation. Both stroke and dementia represent major public health problems. Evidence from epidemiological studies indicates that their risk profiles have many similarities (see Table 1), so integrated intervention strategies will be required for effective prevention

\section{Common Lifestyle-related Factors in Stroke and Dementia}

\section{Obesity}

General obesity, i.e increased body mass index (BMI) and abdominal obesity (increased waist to hip ratio), have been reported as independent risk factors for stroke (even after controlling for hypertension, diabetes and cholesterol levels). ${ }^{1}$ The association between obesity and dementia/AD risk has been less studied. Weight loss seems to occur during the pre-clinical phases of dementia, and recent follow-up studies, e.g. Personnes Agées Quid (PAQUID) and the Honolulu Asia Ageing Study, suggested that low BMI could actually be an early sign of dementia. There is increasing evidence from long-term population-based studies, e.g. the Cardiovascular Risk Factors, Ageing and Dementia (CAIDE) study and the Kaiser Permanente Study, that high BMI in mid-life or late life nine to 18 years prior to dementia (after the Gothenburg study) is associated with increased $A D$ risk. 6,7 Obesity is related to vascular disorders, which could be the link to dementia/AD. For example, obesity is an essential feature in metabolic syndrome, which is also characterised by dyslipidaemia, hypertension, glucose intolerance and insulin resistance. Metabolic syndrome has been related to an increased risk of both stroke and dementia. ${ }^{8,9} \mathrm{~A}$ higher BMI and waist-to-hip ratio were related to white matter lesions (WML) in some studies, and a higher BMI was associated with greater temporal lobe atrophy and greater brain atrophy rate. ${ }^{6}$ These associations were independent of several vascular factors, suggesting a more direct role of obesity in neurodegeneration. Insulin resistance is one possible factor related to $A D$ pathogenesis. ${ }^{10}$ Furthermore, adipose tissue is an important endocrine organ and secretes hormones and several other bioactive compounds. Other adipose-associated (e.g. sex hormones) and adiposederived factors (e.g. leptin, interleukins, growth factors) may be involved. 
Table 1: Main Proposed Risk and Protective Factors Common for Stroke and Dementia

\begin{tabular}{lll}
$\begin{array}{l}\text { Non-modifiable } \\
\text { Risk Factors }\end{array}$ & Risk Factors & Modifiable Factors \\
\hline Advanced age & Cerebrovascular disease/stroke & High education \\
Genetic factors & Cardiovascular diseases & Physical activity \\
e.g. ApoE 84 & Hypertension & Active lifestyle \\
Family history & Hypercholesterolaemia & Alcohol consumption \\
& Obesity & Antioxidants \\
& Diabetes & Fish oils \\
& Smoking & Antihypertensives \\
& Homocysteine & Statins \\
& Psychosocial stress/depression & \\
\hline
\end{tabular}

Some of these factors have a stronger association with stroke, others with dementia. The results of different studies depend on how the outcomes were defined.

\section{Diet}

Diet, especially fat intake, has a well-known association with coronary heart disease (CHD). However, the relationship with stroke or dementia is less clear. There is a lack of well-designed epidemiological studies addressing the role of dietary fats in different stroke subtypes. ${ }^{11} \mathrm{~A}$ proper outcome definition in such studies is crucial, as atherosclerotic changes may have different causes in different types of cerebral arteries. Atherosclerosis may be involved in large artery infarction, but other factors (e.g. blood pressure) may have more important roles in other types of ischaemic stroke (lacunar and cardioembolic infarction). The pattern for dementia/AD seems to follow the one for CHD. A diet rich in saturated fat and cholesterol may increase dementia/AD risk: polyunsaturated fatty acids and fish may be protective. However, contradictory findings exist as well. ${ }^{12}$ The studies conducted so far had a relatively short follow-up, and are therefore prone to bias due to subclinical dementia (i.e. poor diet could be a consequence rather than a cause of disease), and long-term prospective studies are still needed to clarify the matter. Recent data from the CAIDE study indicate that moderate intake of unsaturated fats at mid-life is protective, whereas moderate intake of saturated fats may increase the AD risk, especially among apolipoprotein E (ApoE) $\varepsilon 4$ carriers. ${ }^{13}$

There are several pathways through which fat intake may be related to AD. A high saturated fat intake is linked to vascular factors and oxidative stress. Unsaturated fatty acids may confer protection through their anti-inflammatory properties. Fatty acids may be important in the synthesis and fluidity of nerve cell membranes for synaptic plasticity and neuronal degeneration. Experimental studies using transgenic mouse models of $A D$ have shown that essential omega-3 fatty acids protect against neuronal deficits, decrease $\beta$-amyloid levels and decrease the number of activated microglia in the brain. ${ }^{14,15} \mathrm{In}$ a recent randomised controlled trial, omega-3 fatty acid supplementation did not influence cognitive functioning during a follow-up of six months. However, positive effects were observed in a small group of patients with very mild AD (Mini-Mental State Examination [MMSE] >27). ${ }^{16}$ Therefore, omega-3 may have a role in the primary and possibly the secondary prevention of the disease, but not when the disease process has already taken over.

Oxidative stress is important in both stroke and dementia/AD, and some studies (but not all) suggested that a diet rich in antioxidants might be protective. ${ }^{12,17}$ Low $B_{12} /$ folate levels and their marker, high homocysteine, have been linked with an increased risk of both stroke and dementia/
AD. ${ }^{17}$ Many studies focus on individual dietary components, but the effects of single dietary factors may vary depending on other components of the diet, and dietary patterns may have more important influences on risk levels. The 'Mediterranean diet' seems to protect against dementia/AD, even after controlling for several vascular factors. ${ }^{18} \mathrm{~A}$ similar association was found for stroke. ${ }^{17}$

\section{Physical Exercise and Active Lifestyle}

Some shorter-term longitudinal studies indicated an inverse association between regular and high-intensity leisure time physical activity, or some specific form of physical activity (e.g. dancing, walking) and dementia/AD risk, whereas others found no association. ${ }^{19}$ The CAIDE study showed that regular leisure time physical activity in mid-life may protect against dementia and $A D$ later in life. The risk reduction was 50\% for dementia and $60 \%$ for $A D$, even after controlling for several vascular and lifestylerelated factors. ${ }^{20}$ This association was more pronounced among ApoE $\varepsilon 4$ carriers. Physical activity such as leisure time and commuting seems to be protective against stroke as well. ${ }^{1}$ Physical activity is important in promoting general and vascular health, but it may confer its effects through other mechanisms as well, promoting brain plasticity, ${ }^{21}$ thus affecting several gene transcripts and neurotrophic factors relevant for maintenance of cognitive functions. ${ }^{22,23}$ Social and mental activities have also been suggested to protect against dementia/AD. ${ }^{19}$ Generally, an active lifestyle may increase cognitive reserve, ${ }^{24}$ reduce stress and protect against the development or expression of dementia.

\section{Smoking and Alcohol}

The risk of ischaemic stroke increases proportionally to the number of cigarettes smoked per day. ${ }^{25}$ Passive smoking also increases the risk of ischaemic stroke, and smoking cessation has a strong effect on strokerisk reduction. Earlier case-control studies suggested that smoking might protect against AD. However, recent prospective studies indicated that smoking may result in modestly increased risk of dementia/AD. ${ }^{26}$ Results from case-control studies may be biased due to selective survival: smoking is associated with increased mortality among patients with dementia, so this group may be under-represented in cross-sectional samples. ${ }^{27}$ Smoking may increase the risk of dementia/AD through its negative vascular effects, but other factors (increased oxidative stress, inflammation) may also be involved. Heavy alcohol drinking is a risk factor. A J- or U-shaped relation between alcohol drinking and stroke or dementia/AD has been described, suggesting that light to moderate alcohol use may have a protective effect. ${ }^{1,28}$ Several mechanisms lie behind these associations. Alcohol may have beneficial effects on several cardiovascular risk factors, including hypertension, lipid and lipoprotein levels and inflammatory and haemostatic factors, and moderate alcohol drinking has been related to a reduced risk of cardiovascular diseases. Moderate alcohol drinking has been associated with fewer brain infarcts, and a U-shape relationship with WML has been described. ${ }^{29}$ However, excessive alcohol drinking has clear detrimental effects, and even light drinking levels have been related to increased brain atrophy. ${ }^{30}$

Apart from the effect of alcohol per se, other social and lifestylerelated factors associated with certain drinking habits may lie behind the favourable association between moderate alcohol drinking, stroke and cognitive functioning, e.g. people who drink moderately tend to have a generally healthier lifestyle. At the moment, it is not possible to define a specific level of alcohol intake beneficial for brain health. 
Table 2: Cardiovascular Risk Factors, Ageing and Dementia Risk Score

\begin{tabular}{|c|c|c|}
\hline \multicolumn{2}{|c|}{ Risk Factor } & \multirow{2}{*}{$\frac{\text { Points }}{0}$} \\
\hline Age & $<47$ years & \\
\hline & $47-53$ years & 3 \\
\hline & $>53$ years & 4 \\
\hline \multirow[t]{3}{*}{ Education } & $>10$ years & 0 \\
\hline & $7-9$ years & 2 \\
\hline & $<9$ years & 3 \\
\hline \multirow[t]{2}{*}{ Sex } & Female & 0 \\
\hline & Male & 1 \\
\hline \multirow[t]{2}{*}{ Blood pressure } & $<140 \mathrm{mmHg}$ & 0 \\
\hline & $>140 \mathrm{mmHg}$ & 2 \\
\hline \multirow[t]{2}{*}{ Body mass index } & $<30 \mathrm{~kg} / \mathrm{m}^{2}$ & 2 \\
\hline & $>30 \mathrm{~kg} / \mathrm{m}^{2}$ & 0 \\
\hline \multirow[t]{2}{*}{ Total cholesterol } & $<6.5 \mathrm{mmol} / /$ & 0 \\
\hline & $>6.5 \mathrm{mmol} / /$ & 2 \\
\hline \multirow[t]{2}{*}{ Physical activity } & Yes & 0 \\
\hline & No & 1 \\
\hline \multicolumn{2}{|c|}{ Total Score } & Dementia Risk (\%) \\
\hline \multicolumn{2}{|c|}{$0-5$} & 1 \\
\hline \multicolumn{2}{|c|}{$6-7$} & 1.9 \\
\hline \multicolumn{2}{|c|}{$8-9$} & 4.2 \\
\hline \multicolumn{2}{|c|}{$10-11$} & 7.4 \\
\hline \multicolumn{2}{|c|}{$12-15$} & 16.4 \\
\hline
\end{tabular}

Taking into account the well-known harm related to excessive alcohol drinking, it may be too early to recommend non-drinkers to start consuming alcohol to protect their brain.

\section{Gene-Environment Interactions}

ApoE is one of the most frequently studied genes in ischaemic stroke, but studies have so far given conflicting results. The ApoE $\varepsilon 4$ allele has been linked to an increased risk of stroke and also to an increased infarct size in some studies, but its exact role in stroke is still uncertain. ${ }^{31}$ To date, $A p o E \varepsilon 4$ is the only genetic risk factor for $A D$ of established general significance. ApoE $\varepsilon 4$ is a susceptibility gene for $A D$, being neither necessary nor sufficient for $A D$ development. The mechanisms relating ApoE $\varepsilon 4$ to AD are not completely understood. ApoE has a central role in lipid metabolism: the ApoE $\varepsilon 4$ allele is associated with increased serum total and low-density lipoprotein cholesterol levels, atherosclerosis and $C H D$, but the effect for $A D$ seems to be at least partly independent of these factors. ${ }^{32}$ ApoE has been linked to all major features in $A D$ pathogenesis, e.g. $\beta$-amyloid generation and clearance, neurofibrillary tangle formation, oxidative stress, apoptosis, dysfunction in lipid transport and homeostasis, modulation of intracellular signalling and synaptic plasticity. ${ }^{33}$ Recent epidemiological studies suggest that ApoE $\varepsilon 4$ carriers may be more vulnerable to environmental factors, e.g. physical inactivity, saturated fat intake, alcohol drinking, diabetes, high blood pressure and low $B_{12}$ /folate, and thus could benefit more from lifestyle changes and pharmacological interventions. 13,20,28

\section{Risk Prediction in Stroke and Dementia}

Practical tools are already available for predicting the risk of cardiovascular outcomes, e.g. the Framingham score, diabetes and stroke, but the dementia field lags behind other areas in this regard. Based on data from the CAIDE study, we have recently developed the first dementia risk score including easily measurable variables in mid-life (variables also used in stroke risk scores), which predicts dementia risk two decades in the future (see Table 2). ${ }^{34}$ The CAIDE Dementia Risk Score has been validated in a large $(n=9,831)$, diverse ( $20 \%$ non-Caucasian) population of members of a healthcare delivery system in the US (Kaiser Permanente). ${ }^{35}$ The risk score provides a quantitative estimation of the probability of becoming demented, but it cannot definitively state whether someone will develop dementia. Therefore, it should be used mainly to target preventative measures to those most at risk and not to place labels on individuals. This first version will be further developed by adding new variables, but it can already be used as an educational tool to demonstrate the role of modifiable factors in dementia.

\section{Conclusions}

The brain needs to be thought of as an end-organ. A 'network brain' approach would be more appropriate than the classic 'stroke brain' approach. Brain diseases are different from one another, but it has to be remembered that 'brain failure' occurs through common basic pathophysiological mechanisms. For example, oxidative stress, excitotoxicity, mitochondrial DNA damage, impaired neurotransmitter release and apoptosis underlie not only stroke and $A D$, but also Parkinson's disease, epilepsy, amyotrophic lateral sclerosis, multiple sclerosis and other brain disorders. ${ }^{5}$ Perhaps cardiology is several years ahead with regards to epidemiological studies showing that reducing risk factors through pharmacological treatment and lifestyle changes can reduce CHD risk. Such longitudinal studies need to become integrative, to look beyond the heart and into the brain. Minding heart health may protect the brain as well, but current clinical guidelines for cardiovascular diseases may need to be refined once cognitive aspects are taken into account in order to formulate effective prevention strategies.
1. Hankey GJ, Stroke, 2006;37:2181-8.

2. Aguero-Torres $H_{1}$ Ann N Y Acad Sci, 2000; 903:547-52

3. Pasquier $F$, Leys $D$, Scheltens $P$, I Neural Transm Suppl, 1998;54:117-27.

4. Aguero-Torres $H$, Kivipelto $M$, von Strauss $E$, Dement Geriatr Cogn Disord, 2006;22:244-9.

5. Hachinski V, Stroke, 2007;38:1396-1403.

6. Gustafson D, Lancet Neurology, 2006;5:713-20.

7. Kivipelto M, Ngandu T, Fratiglioni L, et al., Arch Neurol, 2005:62:1556-60.

8. Solenski NJ, Current Drug Targets, 2007;8:802-16.

9. Razay G, Vreugdenhil A, Wilcock G, Arch Neurol, 2007;64: 93-6.

10. Qiu WQ, Folstein MF, Neurobiol Aging, 2006;27:190-98.

11. He K, Xu J, van Horn L, J Am Diet Assoc, 2007; 107:287-95.

12. Luchsinger JA, Mayeux R, Lancet Neurology, 2004;3(10):579-87.

13. Laitinen M, Ngandu T, Rovio S, et al., Dement Ger Cogn Disord, 2006;22:99-107.
14. Calon F, Lim GP, Yang F, et al., Neuron, 2004;43(5):633-45.

15. Oksman $M$, Livonen $H$, Hogyes $E$, et al., Neurobiol Dis, 2006;23(3):563-72.

16. Freund-Levi $Y$, Eriksdotter-Jonhagen $M$, Cederholm T, et al., Arch Neurol, 2006:63(10):1402-8.

17. Ding EL, Mozaffarian D, Semin Neurol, 2006;26(1):11-23.

18. Scarmeas N, Stern Y, Mayeux R, Arch Neurol, 2006;63(12): 1709-17.

19. Fratiglioni L, Paillard-Borg S, Winblad B, Lancet Neurol, 2004;3(6):343-53.

20. Rovio S, Kåreholt I, Helkala E-L, et al., Lancet Neurol, 2005;4: 705-10.

21. Colcombe SJ, Kramer AF, Erickson KI, et al., Proc Natl Acad SC U S A, 2004;101(9):3316-21.

22. Berchtold NC, Kesslak JP, Cotman CW, J Neurosci Res, 2002;68:511-21.

23. Tong L, Shen H, Perreau VM, et al., Neurobiol Dis, 2001;8: 1046-56.

24. Kramer A, Hahn S, Cohen NJ, et al., Nature, 1999:400:418-19.
25. Romero JR, Current Drug Targets, 2007;8: 794-801.

26. Reitz C, den Heijer T, van Duijin C, et al., Neurology, 2007:69(10):998-1005

27. Fratiglioni L, Wang HX, Behav Brain Res, 2000;113(1-2):117-20.

28. Anttila T, Helkala EL, Viitanen M, et al., BMJ, 2004;329: 539-42.

29. Mukamal KJ, Longstreth WT Jr, Mittleman MA, et al., Stroke, 2001;32(9):1939-46.

30. Ding J, Eigenbrodt ML, Mosley TH Jr, et al., Stroke, 2004;35(1): 16-21.

31. Tonk M, Haan J, J Neurol Sci, 2007;257:273-9.

32. Kivipelto M, Helkala E-L, Laakso MP, et al., Ann Intern Med, 2002;137:149-55.

33. Cedazo-Minguez A, Cowburn RF, J Cell Mol Med, 2001;5: 254-66.

34. Kivipelto $M$, Ngandu T, Laatikainen $T$, et al., Lancet Neurology, 2006:9:735-41.

35. Whitmer RA, Barnes D, Kivipelto M, et al., Alzheimers Dement, 2007:3(3)(Suppl. 2):S170. 


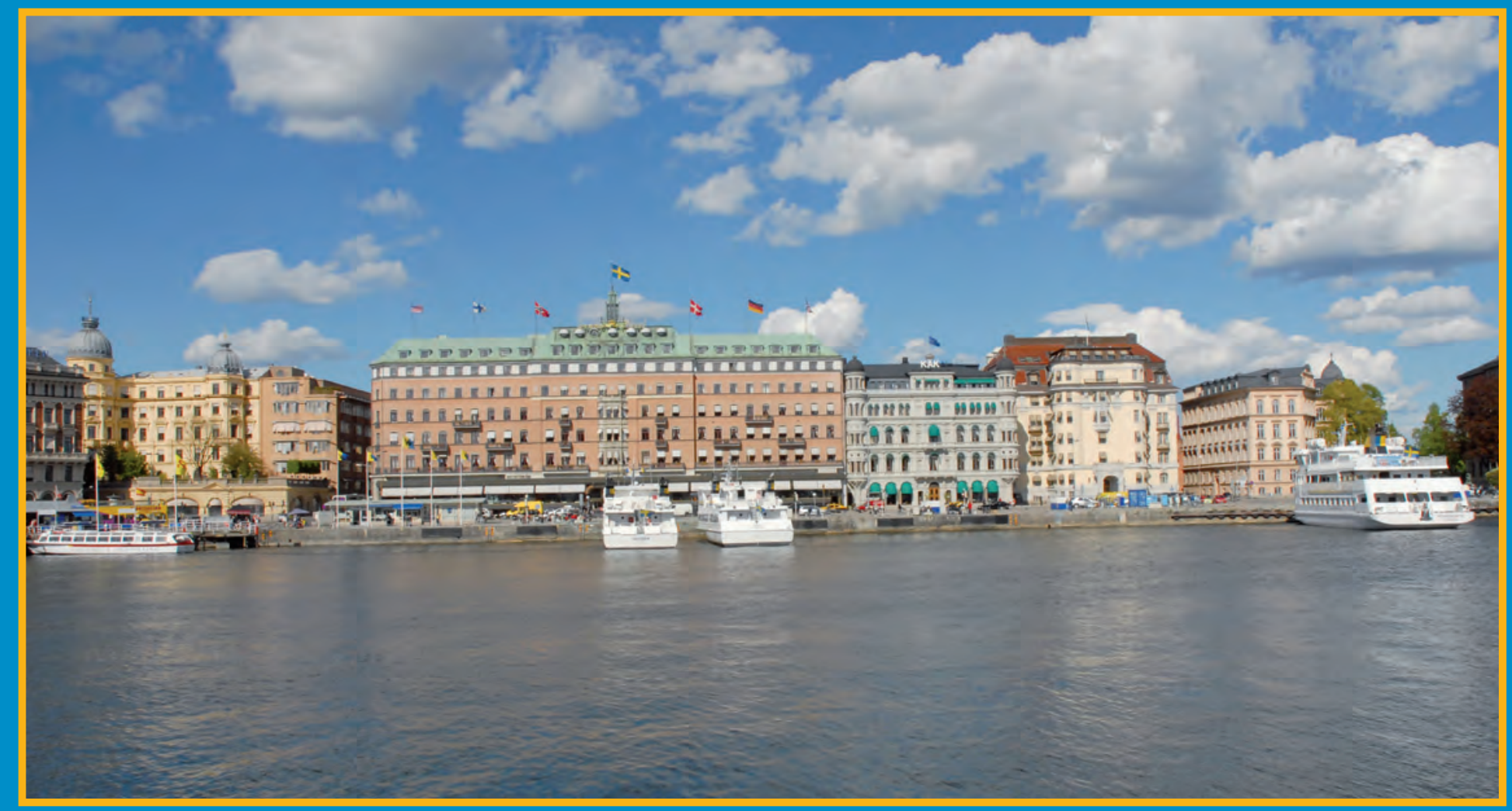

XVIII European Stroke Conference

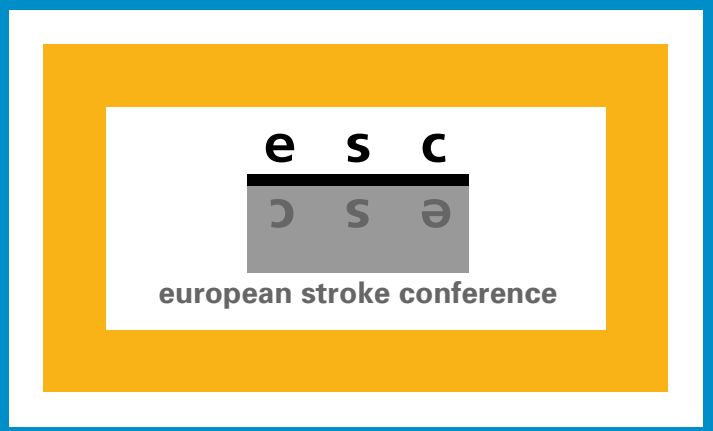

\section{Stockholm, Sweden \\ 26 - 29 May 2009}

www.eurostroke.eu

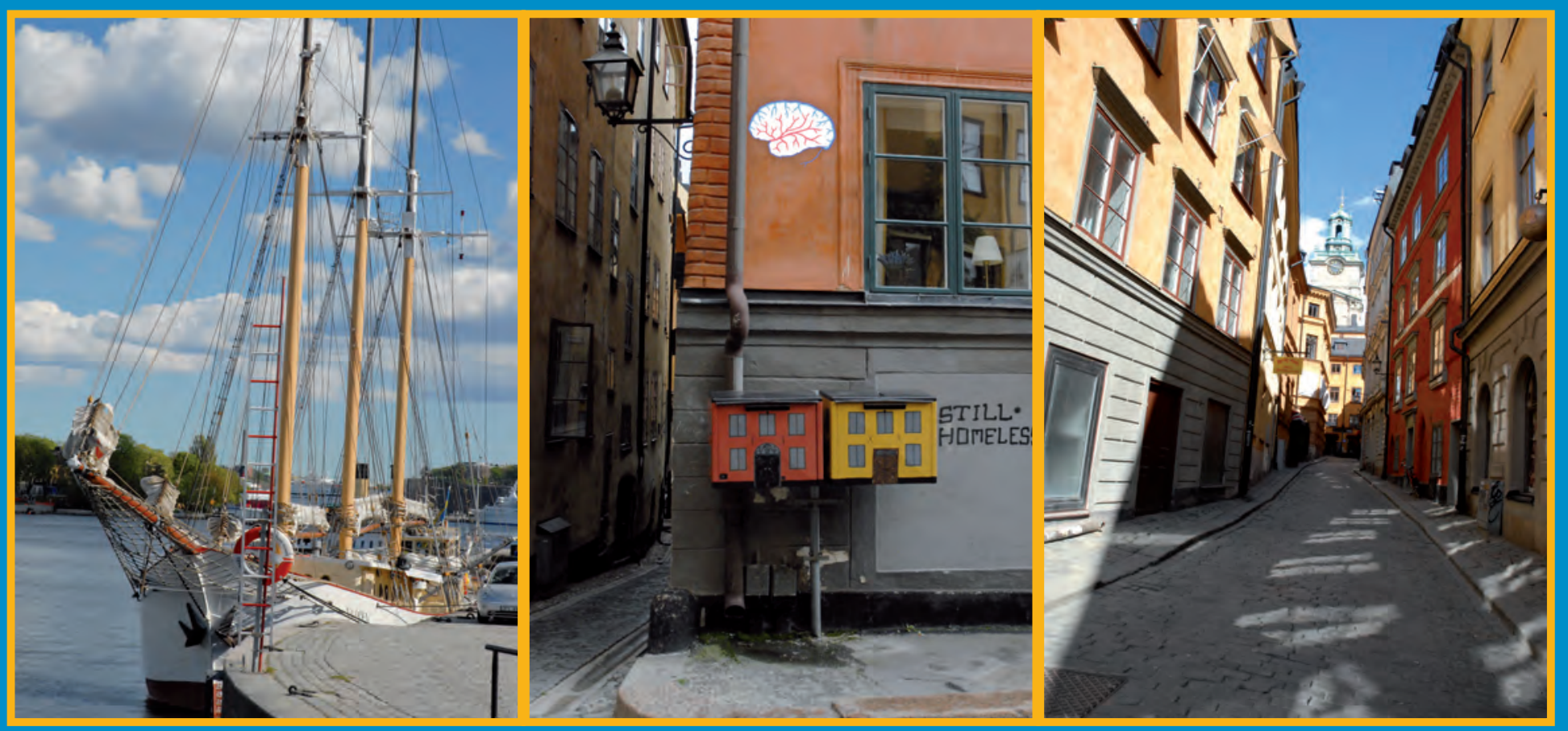

